\title{
Scientific Practices: Equalizing Opportunities for Linguistically Diverse Student Groups
}

\author{
Shelly N. Belleau*广 and Valerie K. Otero ${ }^{\dagger}$ \\ *Longmont High School, Longmont, Colorado, 80501 \\ 'University of Colorado, Boulder, Colorado, 80309
}

\begin{abstract}
This study explores the hypothesis that curricula designed to engage students in evidence-based inductive reasoning can equalize opportunities for linguistically diverse students. Specifically, we evaluated how English language learners and native English speakers performed on conceptual physics assessments and the extent to which they used models and evidence to justify claims and ideas. Results indicate that within this learning context, all students demonstrated conceptual learning gains as well as reliance on evidence and models to support their claims. Female English language learners, a group that has remained underrepresented in science, used evidence and models most frequently to support their claims, and these students demonstrated performance on end of course assessments of conceptual understanding that was comparable to university students. Male English language learners did not rely on evidence and models to the same extent. We discuss these differences and propose rationale for aspects of the learning environment that may have led to these findings.
\end{abstract}

Keywords: Classroom Culture, Scientific Practices, Inquiry Learning, Language

PACS: 01.40.G-, 01.40.-d, 01.55.+b, 01.40.Ha, 43.71 Sy

\section{INTRODUCTION}

For the past 30 years, the PER community has reported that student conceptual understanding and attitudes about science are impacted by instruction that actively engages students and builds on their prior knowledge [1,2]. Traditional methods of teaching science, in which students are asked to accept ideas from a person of authority, have demonstrated lower conceptual learning gains than approaches that engage students in questioning, experimentation and discussion $[1,3,4]$. We suggest that these traditional instructional approaches can lead to students feeling alienated from the classroom community. Inclusive learning environments that shift some authority from the instructor to the students can increase student access to science discourse and practices $[5,6]$.

We propose that a shift in mindset also needs to occur, away from the idea that students learn in spite of the complexity of science and towards the idea that science and its practices can serve as a mechanism that provides meaningful access to classroom discourse for all students, especially those learning English.

The process of scientific induction provides opportunities for students to engage in discussions involving shared observations, explanations, models, and consensus. In this environment, all students, including English language learners, are invited into the learning community and provided an environment to learn science and English [7, 8, 9].
Hispanics are largely underrepresented in the STEM workforce, which has been traditionally composed of white or Asian males [10]. While the nation has observed an increase in the percentage of women in the STEM workforce, minority women continue to be the most underrepresented group. In $2006,26 \%$ of scientists were women, $69 \%$ were white and only $11 \%$ were Hispanic or African American [10]. Students from populations that are traditionally underrepresented in science often must rely on schooling or other sources outside the home to provide positive science experiences [11]. Unfortunately, it is likely that these students take courses that misrepresent science as a set of facts and symbols to be memorized and computed. We hypothesize that by providing these students with opportunities to experience scientific induction, we can greatly increase their identification with science and participation in classroom discourse about scientific investigations. This may lead to higher performance in science class and a greater motivation to pursue science careers.

In this study, we explore this hypothesis by providing students learning the English language (hereafter referred to as ELL students or ELLs) with multiple opportunities to engage in scientific induction using the Physics and Everyday Thinking curriculum [3]. We evaluate the role of gender and ELL status on student use of scientific practices and we discuss classroom structures that could impact how students gain access to science content and practices. 


\section{Science Practices and English Language Learners}

Studies have suggested that both content learning and language acquisition occur in science courses when students have purposeful interactions with peers in an inquiry-based learning environment $[7,8,9,10]$. Environments that engage students in some kind of inquiry can result in inclusive learning opportunities for students, especially for students developing language skills $[7,8]$. Because science, by nature, is collaborative and involves construction and revision of models, we suggest that science is the ideal situation for students learning English to thrive. Physics in particular might be most effective because its study involves shared observations of tangible objects that can be held, pointed to, and discussed, even when the learner is struggling with the English language. Such environments provide multiple modalities for students to engage in discourse and for English language learners to gain access to science.

In this study we investigate two research questions about this learning context: (1) How frequently do students rely on evidence and models to support their claims? (2) Are there gender differences in the way students make or support claims and does ELL status make a difference?

\section{RESEARCH CONTEXT}

Fifty-three $12^{\text {th }}$ grade physics students participated in this study. These students were enrolled at a small urban public high school where $66 \%$ of students qualified for free or reduced lunch programs. The ethnic diversity of the sample was $45 \%$ white and $45 \%$ Hispanic and the gender diversity was $68 \%$ female and $32 \%$ male. Of the 53 students studied, $22 \%(n=12)$ were classified as ELLs by the school system and these students identified that they did not speak English in their homes. In this study, we call these students English Language Learners (ELLs). The first author was the teacher of this course.

\section{Physics and Everyday Thinking}

Student learned physics in a year-long course with Physics and Everyday Thinking (PET), an inquirybased physics curriculum that is designed to engage students in inductive reasoning and follows a guided scientific model-building approach. PET is based on five design principles drawn from sociocultural and cognitive research that allow classrooms to become inclusive learning environments where students develop, share, critique, and revise ideas [12].

PET was originally designed to be a semester-long curriculum for use with non-science majors in university settings. In our previous implementations in the urban high school setting, we found that students demonstrated conceptual understanding on the PET post-test that was comparable to university students and the learning context fostered student tendencies to ask questions and engage in argumentation [13].

Student use of scientific practices, including interpreting evidence and building and applying models, was one of the primary goals in this physics course. Students defended and revised their models during small-group and whole-class discussions. Since this learning environment fostered shared observations and opportunities for students to solve perplexing issues stemming from their observations, we predicted that ELLs would perform as well on a conceptual assessment as native English speakers.

\section{METHODOLOGY}

Our goal was to examine students' performance on a conceptual assessment and the extent to which students used evidence and models and to make and defend scientific claims. Two analyses were done, a pre-post analysis of the normalized gain [4] on the PET conceptual test and a qualitative frequency analysis of student use of claims and of evidence and models as rationale to support their claims.

\section{Conceptual Understanding and Growth}

To evaluate concept understanding and growth, we administered the PET pre- and post-test at the beginning of the course (August) and end of the course (May). The concept test consisted of nine questions that addressed content from the course, including energy, forces, circuits, and light.

We scored student responses using the scoring rubric and sample responses provided with the curriculum. We compared student scores to normative PET post-test data from twenty-seven semester-long university classes [3]. We also examined the average normalized gain (g-ave) for four subgroups: native English speakers (male and female) and ELLs (male and female).

\section{Use of Evidence and Models}

To evaluate student use of models and evidence, we examined their responses to nine open-ended questions on the cumulative final exam, administered at the same time as the PET post-test. These questions covered content from each of the six PET chapters by asking students to write a scientific explanation for a given phenomenon. Students were not prompted to use models or evidence in their responses, however, these scientific practices were modeled for students throughout the course.

We considered a "claim" to be a statement that responded to the given assessment question, therefore each question was only coded as having one claim. 
For the purposes of this study, we defined evidence as an observation used to justify a claim. Evidence could have originated from either a shared observation in class (such as a laboratory finding) or a personal experience outside of class. We defined models as tools that are used to explain why or how a set of phenomena occurs, or to describe a relationship, including a mathematical relationship (formula or written explanation), a graphical representation, or a visual depiction (i.e. force, energy, or light-ray diagrams). Table 1 provides examples of each code.

TABLE 1. Examples of Codes

\begin{tabular}{l|l}
\hline \multicolumn{1}{c|}{ Evidence-Based Rationale } & \multicolumn{1}{|c}{ Model-Based Rationale } \\
\hline $\begin{array}{l}\text { In magnetism it is more } \\
\text { reasonable to use N/S with } \\
\text { models because magnets line } \\
\text { up with the geographical } \\
\text { north/south when floated or on } \\
\text { a pivot. }\end{array}$ & $\begin{array}{l}\text { The heavier object will have } \\
\text { more gravitational pull than } \\
\text { the lighter one. }\end{array}$ \\
$\begin{array}{l}\text { The block, moving already to } \\
\text { the left, will slow down and } \\
\text { then continue to increase in } \\
\text { speed. In a lab we did, when a } \\
\text { fan force on a car was the } \\
\text { opposite direction as the } \\
\text { motion the car, the car slowed } \\
\text { down and then moved in the } \\
\text { opposite direction increasing at } \\
\text { a constant rate. }\end{array}$ & $\begin{array}{l}\text { Because when a object is } \\
\text { moving at a constant speed } \\
\text { there is balanced forces acting } \\
\text { upon it, O net force. According } \\
\text { to Newton's second law if an } \\
\text { object had a net force there } \\
\text { would be unbalanced forces } \\
\text { meaning it would either be } \\
\text { slowing down or speeding up. }\end{array}$ \\
\hline Evidence Omitted & Model Omitted \\
\hline $\begin{array}{l}\text { The snow is a white surface, } \\
\text { which means it scatters and } \\
\text { reflects light in all directions. }\end{array}$ & $\begin{array}{l}\text { An object will stay in motion } \\
\text { unless another force acts on it. }\end{array}$ \\
\hline
\end{tabular}

We first examined whether each student's response to the question (claim) was scientifically accurate, independent of whether evidence or a model was provided. We then classified each response based upon four codes indicating whether the claim was justified only with evidence, only a model, with both evidence and a model, or by neither evidence nor a model. We analyzed the extent that student use of rationale in the form of models and evidence corresponded with scientifically accurate claims.

We also analyzed the frequency that each student used these forms of support for their claims on the nine questions. We then calculated the average frequency for the following four subgroups: native English speakers (male and female) and ELLs (male and female).

\section{FINDINGS}

Finding I: Students demonstrated learning gains comparable to university students learning with the same curriculum.

In this study, the mean post-test score was $61 \%$ (SD 0.21) and the average normalized gain was 0.54
(SD 0.24). When implemented in 27 semester-long university courses (typically with undergraduate nonscience majors), the mean post-test score was $54.2 \%$ and normalized gain was 0.4 (SD 0.13) [3]. Average scores and gains for each subgroup are presented in Figure 1.

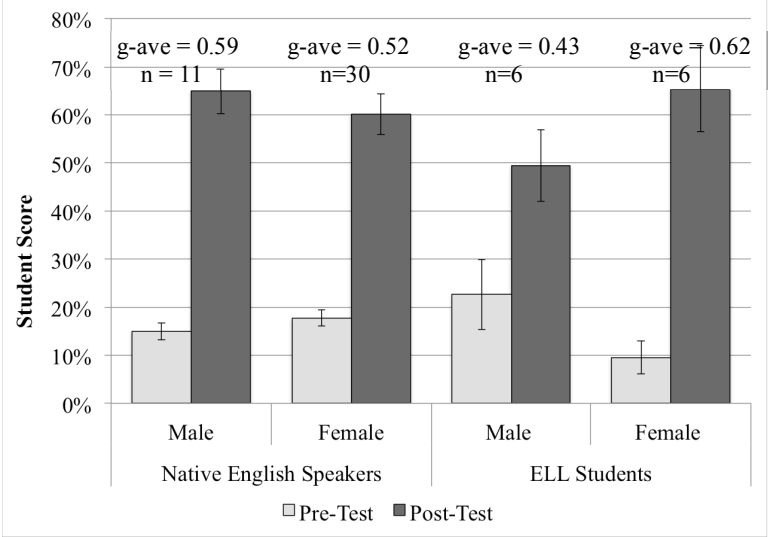

FIGURE 1. Student Pre- and Post-Test Performance

Finding 2: The extent that students supported their responses with evidence or models correlates with the scientific accuracy of the claims.

When students used both evidence and a model to support their claims, $97 \%$ of the claims were scientifically accurate. Conversely, when neither form of rationale was used, only $42 \%$ of the claims were scientifically accurate (Figure 2).

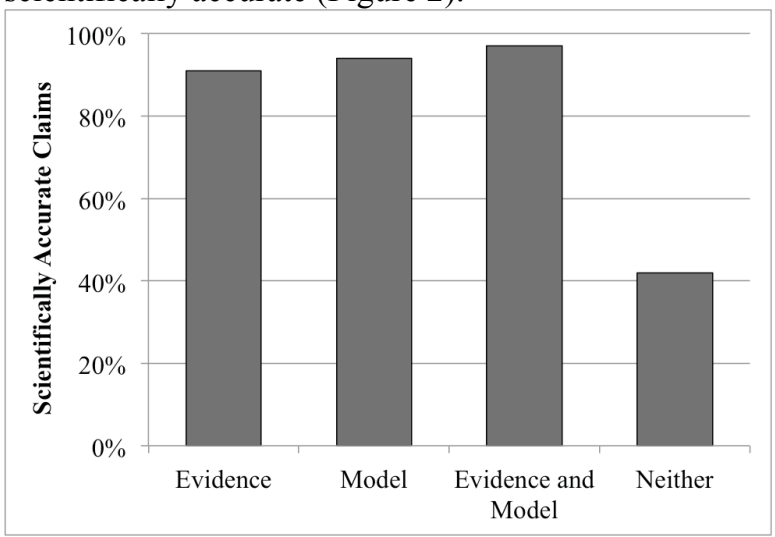

FIGURE 2. Rationale Used and Scientific Accuracy

Finding 3: Female ELLs demonstrated the most frequent use of models and evidence to support their claims

On average, female ELL students supported their claims (with either evidence or a model, or both) on 8 out of the 9 open-ended questions. This compares to 6.4 for native females, 7.1 for native males, and 5.6 for ELL males. ELL females also most commonly used both evidence and a model to support their claims (an average of 5 occurrences, compared to 3.4 for both native male and native female subgroups and 2.8 for ELL males). These findings are presented in Figure 3. 


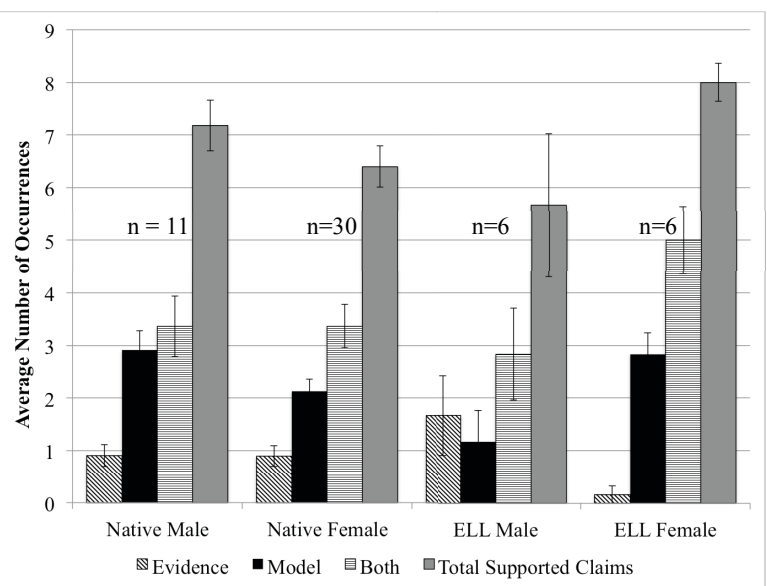

FIGURE 3. Rationale used by Language/Gender Subgroups

\section{CONCLUSIONS}

Female students who are not native English speakers, one of the most underrepresented groups in the field of science, excelled when learning physics in an environment that emphasized the scientific practices of collecting and interpreting evidence and building and applying models. This group of students exhibited the greatest gains on the conceptual assessment and the most frequent use of evidence and models to defend their thinking. We observed these students take risks during whole-class discussions by sharing and revising their ideas in front of their peers. An environment that values emotional risk-taking may be the key to these unusual and desirable gains. However, this idea needs to be more carefully studied in future work.

In this learning environment, it was not only the female ELL students who demonstrated learning gains; their peers displayed conceptual growth that was comparable to university students who learned with the same curriculum. Given that the claims were more correct when using evidence, it could be that centering on practices not only resulted in students becoming proficient in using these practices, but also in their physics understanding.

We propose two key components of this learning environment that impacted student conceptual growth and use of scientific practices: the inclusive classroom culture and the learning environment that was situated in scientific practice.

I. Classroom Culture: In this physics course, the classroom was a safe place for students to take risks and process through ideas as they evolved on the basis of shared experiences. These norms created a learning environment where students felt that their ideas were worth being heard, even when they were struggling with articulating the scientific principle. It was this discourse community that allowed all students to have access to the science ideas and practices.

II. Learning Environment Situated in Practice: Science, by nature, is collaborative and is a social practice. We suggest that the process of students collecting evidence together created shared experiences and attributed to students becoming included within the learning community. In physics this often involves real, tangible objects that can be discussed, pointed to, manipulated, and moved around. This use of real objects, together with a shared problem to investigate, may greatly facilitate English language learners' opportunities and abilities to share their thoughts and participate in the classroom community. Additionally, the scientific practice of building and revising models provides a visual means for students to discuss their ideas. We suggest that this helps equalize the opportunities for students and can be responsible for reducing the language barrier. Inclusion, participation, and a sense of belonging may have a drastic impact on English language learners' future science pursuits.

\section{ACKNOWLEDGEMENTS}

The authors thank the Streamline to Mastery Program, supported by NSF grant DUE 0934921.

\section{REFERENCES}

1. D.E. Meltzer and R.K. Thornton, American Journal of Physics, 80(6) 478-496 (2012).

2. National Research Council, Committee on Undergraduate Physics Education and Implementation, National Academies Press (2013).

3. F. Goldberg, V., Otero, \& S. Robinson, American Journal of Physics, 78(12) 1265-1277 (2010).

4. R.R. Hake, The Physics Teacher, 66(64) 64-74 (1998).

5. S.N. Belleau and V.K. Otero, AIP Conf. Proc., 151311 14 (2013).

6. M.J. Ross, Ph.D. dissertation, University of Colorado at Boulder, 2013.

7. T. Keller, Maine Policy Review, 21(1) 112-119 (2012).

8. R. Mayes and T. Koballa, The Science Teacher, 79(9) 27-34 (2012).

9. A. Flores and K.C. Smith, Journal of Chemical Education, 90, 152-158 (2012).

10. National Research Council (US). Expanding underrepresented minority participation: America's science and technology talent at the crossroads. National Academies Press (2010).

11. R. Larson, K.R. Branscomb, and A.R. Wiley, Family mealtime as a context of development and socialization. Jossey-Bass (2006)

12. Goldberg, Robinson, Otero. New York: It's About Time: 2008.

13. S.N. Belleau, M.J. Ross and V.K. Otero, AIP Conf. Proc., 1413 127-130 (2012). 